\title{
Maternal Hypothyroidism Increases the Risk of Attention-Deficit Hyperactivity Disorder in the Offspring
}

\author{
Darios Getahun, MD, PhD, MPH ${ }^{4,5}$ \\ ${ }^{1}$ Department of Foundations of Medicine, NYU-Long Island School of \\ Medicine, Mineola, New York \\ 2 Department of Obstetrics and Gynecology, NYU-Winthrop Hospital, \\ Mineola, New York \\ ${ }^{3}$ Department of Obstetrics and Gynecology, Kaiser Permanente West \\ Los Angeles Medical Center, Los Angeles, California \\ ${ }^{4}$ Department of Research and Evaluation, Kaiser Permanente \\ Southern California, Pasadena, California \\ ${ }^{5}$ Department of Health Systems Science, Kaiser Permanente \\ Bernard J. Tyson School of Medicine, Pasadena, California \\ Am J Perinatol 2021;38:191-201.
}

Morgan R. Peltier, PhD ${ }^{1,2}$ Michael J. Fassett, MD, FACOG ${ }^{3}$ Vicki Y. Chiu, MS ${ }^{4}$

\author{
Address for correspondence Morgan R. Peltier, PhD, Department of \\ Foundations of Medicine, NYU-Long Island School of Medicine, \\ 101 Mineola Blvd, Suite 04-040, Mineola, NY 11501 \\ (e-mail: morgan.peltier@nyulangone.org).
}

\begin{abstract}
Keywords

- attention-deficit hyperactivity disorder

- ADHD

- hypothyroidism neurodevelopment

- pregnancy

- preterm birth

- thyroid

Objective This study aimed to determine if hypothyroidism prior to, or during, pregnancy increases the risk of attention-deficit hyperactivity disorder (ADHD) in the child and how the association may be modified by preterm birth, sex of the child, and race-ethnicity.

Study Design Data were abstracted from linked maternal-child medical records. Incidence rate differences (IRDs), adjusted hazard ratios (aHRs), and their 95\% confidence intervals ( $\mathrm{Cls}$ ) were estimated to evaluate the association of maternal hypothyroidism with childhood ADHD risk. Stratified analyses were used to evaluate whether the association is affected by timing of first diagnosis, gestational age at birth (term vs. preterm), sex, and race-ethnicity.

Results Hypothyroidism diagnosed prior to $(\operatorname{IRD}=1.30)$, or during $(\operatorname{IRD}=0.59)$ pregnancy increases the risk of ADHD in the children (aHR $=1.27 ; 95 \% \mathrm{Cl}: 1.15$, 1.41 , and $1.17 ; 95 \% \mathrm{Cl}: 1.00,1.38)$. The association was strongest when diagnosed during the first trimester (IRD $=0.97$ and $\mathrm{aHR}=1.28$; $95 \% \mathrm{Cl}: 1.04,1.58)$. For children born preterm, there was significantly increased risk of ADHD if their mothers were diagnosed prior to (IRD $=3.06$ and $\mathrm{aHR}=1.43 ; 95 \% \mathrm{Cl}: 1.09,1.88)$, but not during pregnancy. The effect of maternal hypothyroidism on increased risk of ADHD was stronger for boys ( $I R D=1.84$ and $a H R=1.26 ; 95 \% \mathrm{Cl}: 1.14,1.40)$ than it was for girls $(\mathrm{IRD}=0.48$ and $\mathrm{aHR}=1.19 ; 95 \% \mathrm{Cl}: 1.01,1.40)$ and for Hispanic children $(\mathrm{IRD}=1.60$ and $\mathrm{aHR}=1.45 ; 95 \% \mathrm{Cl}: 1.25,1.68)$ compared with other race ethnicities.

Conclusion Exposure to maternal hypothyroidism during the periconceptual period significantly increases the risk of ADHD and that the association varies with gestational age at delivery, child sex, and race-ethnicity.
\end{abstract}

received

December 20, 2019

accepted

August 22, 2020

published online

October 21, 2020 (c) 2020. Thieme. All rights reserved.

Thieme Medical Publishers, Inc.,

333 Seventh Avenue, 18th Floor,

New York, NY 10001, USA
DOI https://doi.org/

10.1055/s-0040-1717073.

ISSN $0735-1631$. 


\section{Key Points}

- Maternal hypothyroidism increases the risk of ADHD diagnosis in the offspring.

- The association of maternal hypothyroidism with childhood ADHD was influenced by timing of diagnosis.

- Strength of the association was strongest in preterm born infants, boys, and Hispanic children.

Attention-deficit hyperactivity disorder (ADHD), a neurodevelopmental disability affecting $9.4 \%$ of children 2 to 17 years of age, is characterized by difficulties in executive function, inattention, hyperactivity, and impulsivity. ${ }^{1}$ It is the most widespread behavior disorder of children and it increases the risk of other life-long problems such as substance abuse, ${ }^{2}$ relationship problems, ${ }^{3,4}$ poorer job $^{5}$ and academic performance, ${ }^{6}$ riskier driving, ${ }^{5}$ criminality, ${ }^{7}$ and suicide rates. ${ }^{8}$ It is estimated to cost $\$ 143$ to 266 billion in lost wages, increased health, and educational costs. ${ }^{9}$

Many of the genes implicated in childhood ADHD including: dopamine receptor $4,{ }^{10}$ monoamine oxidase, ${ }^{11}$ dopamine $\beta$ hydroxylase, $^{12}$ DOPA decarboxylase, ${ }^{12}$ adiponectin, ${ }^{13}$ and brain-derived neurotrophic factor ${ }^{14}$ are regulated by the thyroid hormones that are critical for various processes in neurodevelopment such as stem cell differentiation, neuron growth, synapse formation, and myelination. Therefore, several researchers have explored the possibility that thyroid dysfunction during pregnancy may be a cause of the condition. However, the results have been conflicting. ${ }^{15-18}$ Thyroid peroxidase autoantibodies in the maternal plasma increased the risk of ADHD-type problems by 1.77 -fold ${ }^{15}$ and severe hypothyroxinemia $(<5$ th percentile) at 12.9 weeks increased the risk of ADHD by 1.70 -fold in a Dutch population. ${ }^{16}$ Others, however, found no association of maternal thyroid peroxidase antibody or fT4 concentrations with risk of ADHD but did report 1.39-fold increased risk of ADHD symptoms in female offspring of women with high thyroid-stimulating hormone (TSH) levels. ${ }^{17}$ A Danish study revealed that maternal hyperthyroidism, not hypothyroidism was associated with a 1.23 fold increased risk of ADHD in the children. ${ }^{18}$ These studies were conducted in regions of Europe where there are nationalized health care systems and ethnically homogeneous populations and the findings may not be generalizable to children born in the United States where the health care is largely privately managed, preterm birth rates are higher, and there is significant racial and ethnic diversity. Different countries have different determinants of disease for both hypothyroidism and ADHD and access to health care may amplify the association. Therefore, effect estimates of a potential relationship between hypothyroidism and ADHD may differ in the United States. Although a previous study, reported an association between maternal hypothyroidism and intellectual disability/inattention in the United States, ${ }^{19}$ it relied on subscores of the Wechsler Intelligence Scale for Children to diagnose the inattention rather than formal diagnosis of ADHD. Furthermore, no potential interactions of hypothyroidism with maternal race/ethnicity, timing of diagnosis, gestational age at birth, or fetal sex were explored. Therefore, we evaluated the risk of ADHD in children born to women with and without hypothyroidism in a large diverse Southern California population and how this potential risk factor is impacted by timing of exposure to the hypothyroidism, gestational age at delivery, child's sex, and race-ethnicity.

\section{Materials and Methods}

\section{Study Population}

This study was approved by the Kaiser Permanente Southern California (KPSC) Institutional Review Board with exempt status. The study population for this retrospective cohort study was drawn from a total population of 571,674 births that occurred between January 1, 2000 and December 31, 2016 ( - Fig. 1).The information used in this study was obtained from the KPSC electronic health records including: perinatal service (obstetrical conditions and procedures, fetal/neonatal outcomes), maternal and child inpatient/outpatient medical care, laboratory and pharmacy records, maternal sociodemographic and behavioral characteristics, child race ethnicity, age, and sex. Parental demographic and maternal medical and obstetric health records were linked to the child medical records using medical record numbers unique for each pregnancy. Inclusion criteria included: (1) children born to KPSC members in all KPSC hospitals between January 01, 2000 and December 31, 2016, and (2) delivered between $28^{0 / 7}$ and $42^{6 / 7}$ weeks' gestation. A total of 130,556 births were excluded due to: multiple births, nonlife births, gestation length $<28$ or $\geq 43$ weeks, leaving KPSC membership prior to age three, noncontinuous enrollment for at least 3 months between 3 and 17 years of age, autism diagnosis, ADHD diagnosed but not treated with medication or undiagnosed ADHD with ADHD medication, or ADHD diagnosis prior to age three but not confirmed at later age. The latter exclusion was important to maintain a cleaner cohort regarding exposure and outcome ascertainment. Impact of these exclusions on patient demographics is shown in - Supplementary Table S1 (available in the online version).

\section{Exposures and Outcomes}

International Classification of Diseases, Ninth Revision, Clinical Modification (ICD-9-CM) codes and/or ICD-10-CM: 243, 244.0, 244.1, 244.2, 244.3, 244.8, 244.9, 245.0, E06.0, E89.0, E03.0, E03.2, E03.3, E03.8, and E.03.9 and laboratory test values, TSH $>4.0 \mu \mathrm{IU} / \mathrm{mL}$ or fT $4 \leq 0.80 \mathrm{ng} / \mathrm{dL}$, were used to identify exposures to hypothyroidism (this is consistent with the 2017 ATA guidelines). Distributions of the hypothyroid diagnostic codes and thyroid laboratory values are summarized in - Supplementary Tables \$2 and S3 (available in the online version). Where possible, we further divided the population of women exposed to hypothyroidism into several categories and subcategories based on timing of diagnosis. This included before and during pregnancy. The "pre-pregnancy" category was further subdivided into 120 to 61 and 60 to 1 days prior to pregnancy and the "in pregnancy" category was subdivided between women diagnosed in first, second, and third trimesters. 
Total Births (2000-2016)

$N=571,674$
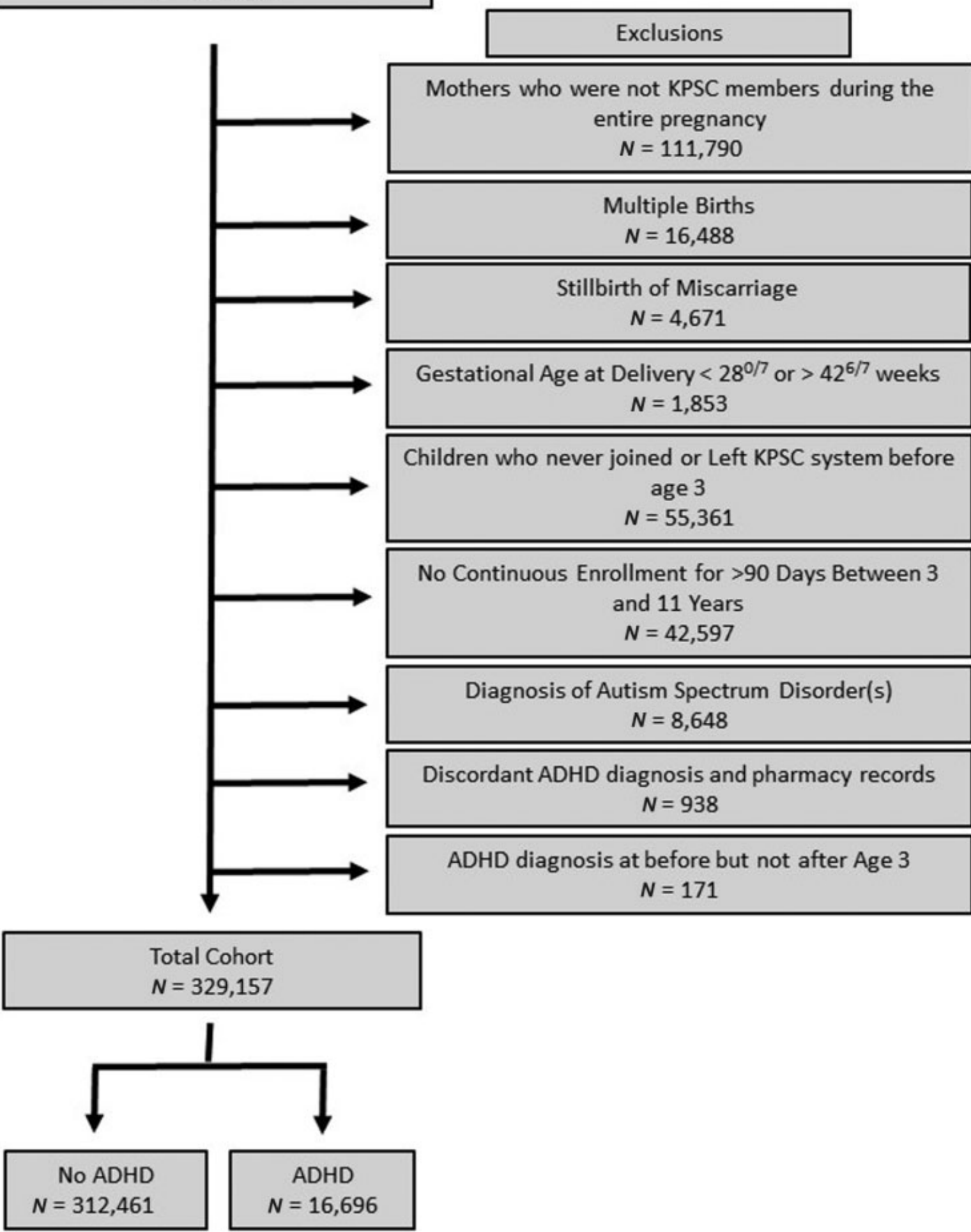

Fig. 1 Flowchart of the decision rules to develop the cohort used for these studies.

Physician diagnosis of ADHD (ICD-9-CM codes 314. $\times$ ) in children 3 to 17 years old on at least one visit during the follow-up period was used to ascertain the outcome variable. In a preliminary analysis, $96 \%$ of children with ADHD were diagnosed by child/adolescent psychiatrists, developmental/ behavioral pediatricians, child psychologists, or neurologists. Follow-up began at birth and ended with ADHD diagnosis or when censoring occurred due to health plan disenrollment, 18th birthday, non-ADHD-related death, or end of the study (December 31, 2016). 


\section{Covariates}

Factors including child sex, race-ethnicity, maternal age, education, median family household income, parity, and timing of prenatal care, smoking habits during pregnancy, and gestational age at delivery were evaluated as potential confounders/effect modifiers. Child race/ethnicity was based on maternal and paternal records and classified as nonHispanic white (White), non-Hispanic black (Black), Hispanic, Asian/Pacific Islander, and other/mixed race/ethnicity.

\section{Statistical Analyses}

Differences between maternal and child characteristics based on children ADHD status were compared using $\chi^{2}$-tests.

Crude and adjusted hazard ratios (aHRs) and their 95\% confidence intervals (CIs) were estimated using Cox proportional hazards models. Stratified analyses were used to determine if the effects of hypothyroidism on ADHD risk may vary due to factors selected a priori that include: timing of hypothyroidism diagnosis, gestational age at delivery, child sex, and race-ethnicity. Because preterm birth may lie on the causal pathway between hypothyroidism and ADHD, we examined the associations with and without adjustment for gestational age at delivery in the model. However, we examined the independent effect of hypothyroidism on ADHD after stratifying the dataset by gestational age (preterm and term births) categories. The categorization of gestational age into term and preterm birth was based on an a priori decision. We also stratified a priori based on sex because males are at increased risk for several neurodevelopmental disorders, including ADHD, that may be due to biological factors. Furthermore, we stratified by race-ethnicity a priori because there are well documented health disparities in minorities that may be due to access to and utilization of health care as well as underlying biological risk factors. Children from women not diagnosed with hypothyroidism were the reference group in all analyses. It is difficult to compare HRs between different strata due to nonlinear effects of the denominator variation on the point estimate. Therefore, we also calculated incidence rates (IRs, defined as the number of cases ADHD divided by the number of person-years) and incidence rate differences (IRDs) with 95\% CIs to compare effect sizes on a linear scale. ${ }^{20}$ Analyses were performed using SAS version 9.4 (SAS Institute, Cary, NC).

\section{Results}

Details of the children with $(n=16,696)$ and without $(n=312,461)$ ADHD diagnosis and their mothers are summarized in - Table 1. Women who gave birth to children with ADHD were younger, tended to finish high school but were more likely to make less than $\$ 30,000$ per year than mothers of non-ADHD children. Mothers of children with ADHD were also more likely to be nulliparous, to deliver preterm, and smoke tobacco during pregnancy.

\section{General Effects of Hypothyroidism}

Of the 329,157 pregnancies followed in this study, 9,675 (2.9\%) had a diagnosis of hypothyroidism $<120$ prior to, or during their pregnancy (319,482 did not). Incidence of ADHD was greater for children born to women with a hypothyroid diagnosis prior to, or during, pregnancy (-Table 2). Coxproportional hazards models also demonstrated that prenatal exposure to hypothyroidism is significantly associated with increased risk of ADHD (aHR:1.24, 95\% CI: 1.14, 1.35) (-Fig. 2; -Table 2). Further analysis by trimester of in utero exposure, revealed that the trend was largely confined to the first trimester (HR: 1.29, 95\% CI: 1.05, 1.59) and that hazard ratios were not affected by adjustment for covariates (-Table 2). Children of women whose hypothyroidism was left either untreated or treated with $<50 \mu \mathrm{g} / \mathrm{d}$ thyroid supplementation were not at significantly increased risk (aHR: $1.08,95 \% \mathrm{CI}: 0.75,1.57)$ of ADHD but children of women whose hypothyroidism was treated with $\geq 50 \mu \mathrm{g} / \mathrm{d}$ were at significantly increased risk (aHR: 1.26, 95\% CI: 1.14, 1.39). Exposure to hypothyroidism was associated with increased risk of ADHD when diagnosed prior to or after conception.

\section{Stratification by Gestational Age at Delivery}

Although no statistical interaction was detected between hypothyroidism and preterm birth $(p=0.439)$, when stratified by the timing of first diagnosis, maternal hypothyroidism was associated with increased incidence and risk of ADHD in preterm born infants with the strongest associations for women first diagnosed during the pre-pregnancy period (aHR: 1.43, 95\% CI: 1.09, 1.88; - Table 3). When further stratified, the association was significantly higher for those diagnosed at 61 to 120 day during pre-pregnancy (aHR: 1.42, 95\% CI: 1.01, 1.99), but not for those diagnosed at 1 to 60 days prior to pregnancy. The IRDs in ADHD for infants born to hypothyroid versus nonhypothyroid mothers were much higher for preterm infants than it was for term infants (-Table 3) and the incidence of ADHD in children born to hypothyroid mothers delivering at term was strongest in the first trimester ( - Table 3 ), similar to what was observed in the unstratified analysis ( - Table 2 ). Adding gestational age of delivery to the model had no effect on the association $(\mathrm{aHR}=1.23,95 \% \mathrm{CI}: 1.13,1.35)$.

\section{Stratification by Sex}

As expected, there were 2.5 times more boys than girls in this study with ADHD (- Table 4). Although a diagnosis of maternal hypothyroidism overall tended to increase the hazard of ADHD in girls, the increased incidence was marginal (aHR: 1.19, 95\% Cl: 1.01, 1.40). For male infants, however, maternal hypothyroidism diagnosed prior to pregnancy (aHR: 1.30 , $95 \%$ CI: $1.15,1.46$ ) or within the first trimester (aHR: 1.28 , 95\% CI: $1.00,1.64)$ was significantly associated with increased incidence and hazard of ADHD. Evaluation of the IRs stratified by sex, suggests that hypothyroidism may have a stronger effect on boys than it does on girls because differences between IRD in children born of hypothyroid mothers and those whose mothers did not have hypothyroidism were four times larger for boys than it was for girls (IRD 1.84 vs. 0.48; - Table 4). Tests for a statistical interaction between sex and hypothyroidism did not reach statistical significance $(p=0.563)$, however. 


\begin{tabular}{|c|c|c|c|}
\hline Characteristics & No ADHD $N=312,461(\%)$ & ADHD $N=16,696(\%)$ & $p$-Value \\
\hline \multicolumn{4}{|l|}{ Maternal age, $y$} \\
\hline$<20$ & $13,783(4.4)$ & $1,020(6.1)$ & \multirow[t]{4}{*}{$<0.001$} \\
\hline $20-29$ & $127,830(40.9)$ & $7,070(42.3)$ & \\
\hline $30-34$ & $101,309(32.4)$ & 4,971 (29.8) & \\
\hline$\geq 35$ & $69,539(22.3)$ & $3,635(21.8)$ & \\
\hline \multicolumn{4}{|l|}{ Maternal education, y } \\
\hline$<12$ & $23,238(7.4)$ & $1,211(7.3)$ & \multirow[t]{4}{*}{$<0.001$} \\
\hline 12 & $82,636(26.4)$ & $4,716(28.2)$ & \\
\hline$\geq 13$ & $202,974(65.0)$ & $10,620(63.6)$ & \\
\hline Missing & $3,613(1.2)$ & $149(0.9)$ & \\
\hline \multicolumn{4}{|c|}{ Median family household income, USD } \\
\hline$<\$ 30,000$ & $21,326(6.8)$ & $1,282(7.7)$ & \multirow[t]{6}{*}{$<0.001$} \\
\hline$\$ 30,000-\$ 49,999$ & $94,995(30.4)$ & $5,164(30.9)$ & \\
\hline$\$ 50,000-\$ 69,999$ & $94,909(30.4)$ & 4,936 (29.6) & \\
\hline$\$ 70,000-\$ 89,999$ & $55,345(17.7)$ & $3,030(18.1)$ & \\
\hline$\geq \$ 90,000$ & $44,705(14.3)$ & $2,180(13.1)$ & \\
\hline Missing & $1,181(0.4)$ & $104(0.6)$ & \\
\hline \multicolumn{4}{|l|}{ Parity } \\
\hline Par 0 & $121,400(38.9)$ & $7,431(44.5)$ & \multirow[t]{4}{*}{$<0.001$} \\
\hline Par 1 & $106,990(34.2)$ & $5,388(32.3)$ & \\
\hline Par 2+ & 84,057 (26.9) & $3,877(23.2)$ & \\
\hline Missing & $14(<0.1)$ & $0(0.0)$ & \\
\hline \multicolumn{4}{|l|}{ Gestational age, wk } \\
\hline $28-36$ & $22,996(7.4)$ & $1,585(9.5)$ & \multirow[t]{2}{*}{$<0.001$} \\
\hline $37-42$ & $289,465(92.6)$ & $15,111(90.5)$ & \\
\hline \multicolumn{4}{|l|}{ Smoking during pregnancy } \\
\hline No & $297,527(95.2)$ & $15,594(93.4)$ & \multirow[t]{2}{*}{$<0.001$} \\
\hline Yes & $14,934(4.8)$ & $1,102(6.6)$ & \\
\hline Late initiation or no prenatal care & $23,137(7.4)$ & $1,233(7.4)$ & 0.867 \\
\hline \multicolumn{4}{|l|}{ Child's race/ethnicity } \\
\hline White & $56,899(18.2)$ & $4,322(25.9)$ & \multirow[t]{6}{*}{$<0.001$} \\
\hline Black & $21,843(7.0)$ & $1,500(9.0)$ & \\
\hline Hispanic & $129,556(41.5)$ & $5,717(34.2)$ & \\
\hline Asian/Pacific islanders & $31,452(10.1)$ & $632(3.8)$ & \\
\hline Other/Multiple & $72,398(23.2)$ & $4,512(27.0)$ & \\
\hline Unknown & $313(0.1)$ & $13(0.1)$ & \\
\hline \multicolumn{4}{|l|}{ Child's sex } \\
\hline Female & $158,212(50.6)$ & $4,761(28.5)$ & \multirow[t]{2}{*}{$<0.001$} \\
\hline Male & $154,249(49.4)$ & 11,935 (71.5) & \\
\hline
\end{tabular}

Abbreviations: ADHD, attention-deficit hyperactivity disorder; USD, United States Dollar.

Note: Statistically significant associations are indicated in boldface.

\section{Stratification by Race-Ethnicity}

Like child sex, race-ethnicity is a significant risk factor for adverse neurodevelopmental outcomes. Incidence of ADHD was highest in White children, followed by Blacks and Hispanics with Asian/Pacific Islander children having the lowest rates of ADHD. Hypothyroidism increased the incidence of ADHD in Whites, Blacks, and Hispanics to varying degrees but not for Asian/Pacific Islander and children of Other/Multiple ethnicities, possibly because of limited numbers of women experiencing hypothyroidism in 


\begin{tabular}{|c|c|c|c|c|c|c|}
\hline \multirow[t]{2}{*}{ Group } & \multirow[t]{2}{*}{$N$} & \multirow[t]{2}{*}{ Person-years } & \multirow[t]{2}{*}{ IR (\%) } & \multirow[t]{2}{*}{ IRD (95\% Cl) } & \multicolumn{2}{|c|}{ HR (95\% confidence intervals) } \\
\hline & & & & & Crude & Adjusted $^{a}$ \\
\hline No Hypothyroidism & 16,171 & $2,963,448$ & 5.46 & 0.00 (Reference) & 1.00 (Reference) & 1.00 (Reference) \\
\hline Hypothyroidism & 525 & 80,260 & 6.54 & $1.08(0.56,1.60)$ & $1.26(1.15,1.37)$ & $1.24(1.14,1.35)$ \\
\hline Untreated & 89 & 14,455 & 6.16 & $0.70(-0.51,1.91)$ & $1.21(0.98,1.49)$ & $1.23(1.00,1.51)$ \\
\hline Treated & 436 & 65,805 & 6.62 & $1.17(0.60,1.74)$ & $1.27(1.15,1.39)$ & $1.24(1.13,1.37)$ \\
\hline$<50 \mu g$ & 28 & 5,465 & 5.12 & $-0.33(-2.29,1.63)$ & $1.11(0.77,1.61)$ & $1.08(0.75,1.57)$ \\
\hline$\geq 50 \mu g$ & 408 & 60,340 & 6.77 & $1.31(0.71,1.90)$ & $1.28(1.16,1.41)$ & $1.26(1.14,1.39)$ \\
\hline Pre-Pregnancy & 377 & 55,792 & 6.76 & $1.30(0.68,1.92)$ & $1.30(1.17,1.44)$ & $1.27(1.15,1.41)$ \\
\hline \multicolumn{7}{|l|}{ Diagnosis timing } \\
\hline 120 to 61 days & 222 & 35,116 & 6.32 & $0.86(0.09,1.64)$ & $1.22(1.06,1.39)$ & $1.19(1.04,1.36)$ \\
\hline 60 to 1 days & 155 & 20,676 & 7.50 & $2.04(1.03,3.05)$ & $1.43(1.22,1.68)$ & $1.40(1.19,1.64)$ \\
\hline \multicolumn{7}{|l|}{ Treatment status } \\
\hline Untreated & 32 & 4,778 & 6.70 & $1.24(-0.86,3.34)$ & $1.42(1.00,2.00)$ & $1.41(0.99,1.99)$ \\
\hline Treated & 345 & 51,014 & 6.76 & $1.31(0.66,1.95)$ & $1.29(1.16,1.43)$ & $1.26(1.13,1.40)$ \\
\hline$<50 \mu g$ & 22 & 3,489 & 6.31 & $0.85(-1.60,3.30)$ & $1.33(0.87,2.02)$ & $1.32(0.87,2.00)$ \\
\hline$\geq 50 \mu g$ & 323 & 47,525 & 6.80 & $1.34(0.67,2.01)$ & $1.28(1.15,1.43)$ & $1.25(1.12,1.40)$ \\
\hline In-pregnancy & 148 & 24,468 & 6.05 & $0.59(-0.34,1.52)$ & $1.17(0.99,1.37)$ & $1.17(1.00,1.38)$ \\
\hline \multicolumn{7}{|l|}{ Diagnosis timing } \\
\hline First trimester & 91 & 14,158 & 6.43 & $0.97(-0.25,2.19)$ & $1.29(1.05,1.59)$ & $1.28(1.04,1.58)$ \\
\hline Second trimester & 27 & 5,322 & 5.07 & $-0.38(-2.37,1.60)$ & $0.94(0.64,1.37)$ & $0.93(0.64,1.36)$ \\
\hline Third trimester & 30 & 4,988 & 6.01 & $0.58(-1.49,2.61)$ & $1.10(0.77,1.57)$ & $1.15(0.80,1.64)$ \\
\hline \multicolumn{7}{|l|}{ Treatment status } \\
\hline Untreated & 57 & 9,677 & 5.89 & $0.43(-1.04,1.91)$ & $1.12(0.87,1.46)$ & $1.15(0.88,1.49)$ \\
\hline Treated & 91 & 14,791 & 6.15 & $0.70(-0.50,1.89)$ & $1.20(0.98,1.47)$ & $1.19(0.97,1.46)$ \\
\hline$<50 \mu g$ & 6 & 1,976 & 3.04 & $-2.42(-5.68,0.84)$ & $0.69(0.31,1.54)$ & $0.66(0.30,1.46)$ \\
\hline$\geq 50 \mu g$ & 85 & 12,815 & 6.63 & $1.18(-0.11,2.46)$ & $1.27(1.02,1.57)$ & $1.26(1.02,1.56)$ \\
\hline
\end{tabular}

Abbreviations: IR, incidence rates per 1000 person-years; IRD, Incidence Rate Difference, HR, hazard ratio; $\mathrm{Cl}$, confidence intervals. Note: Statistically significant associations are indicated in boldface.

${ }^{a}$ Adjustments were made for maternal age, education, median family household income, smoking during pregnancy, prenatal care, parity, child's sex and race/ethnicity.

these groups ( - Table 5 ). Formal tests for a statistical interaction with the Cox-proportional hazards models were marginally nonsignificant ( $p=0.055)$.

\section{Discussion}

We found that maternal hypothyroidism during the preconceptual and prenatal period significantly increases the hazard of ADHD in the children and that this association is strongest for boys, those born prematurely, and for Hispanic children. IRs for ADHD were generally higher for children born to hypothyroid women regardless of race-ethnicity, sex, timing of birth, or timing of in utero hypothyroid exposure with most of the exceptions being for children of women diagnosed in mid-late pregnancy. The lack of association of maternal hypothyroidism diagnosed later in pregnancy could be due to the sparseness of the data in that category. It is also possible, however, that maternal hypothyroidism has more severe effects on fetal brain development if it occurs prior to 18 weeks gestation when the fetus starts making its own thyroid hormones. ${ }^{21}$

Consistent with previous studies, ${ }^{22,23}$ we found that preterm birth is associated with increased incidence of ADHD in the offspring. Although we did not detect an interaction in the statistical models, maternal hypothyroidism had a stronger effect on incidence of ADHD in preterm $(I R D=2.23)$ than term $(I R D=0.93)$ infants. Disordered neurodevelopmental patterns caused by the lack of thyroid hormones may make the brain more susceptible to injury as a result of the preterm birth. Thyroid hormone supplementation reduced brain damage in animal models of traumatic brain injury and stroke. ${ }^{24}$ Maternal hypothyroidism also increases the risk of preterm birth ${ }^{25,26}$ and it is possible that the correlation between these outcomes is due to the fact that infants who are at risk for ADHD because of maternal hypothyroidism are also at increased risk for preterm birth. Our finding is that the risk for ADHD remained elevated when models were adjusted for gestational age at 


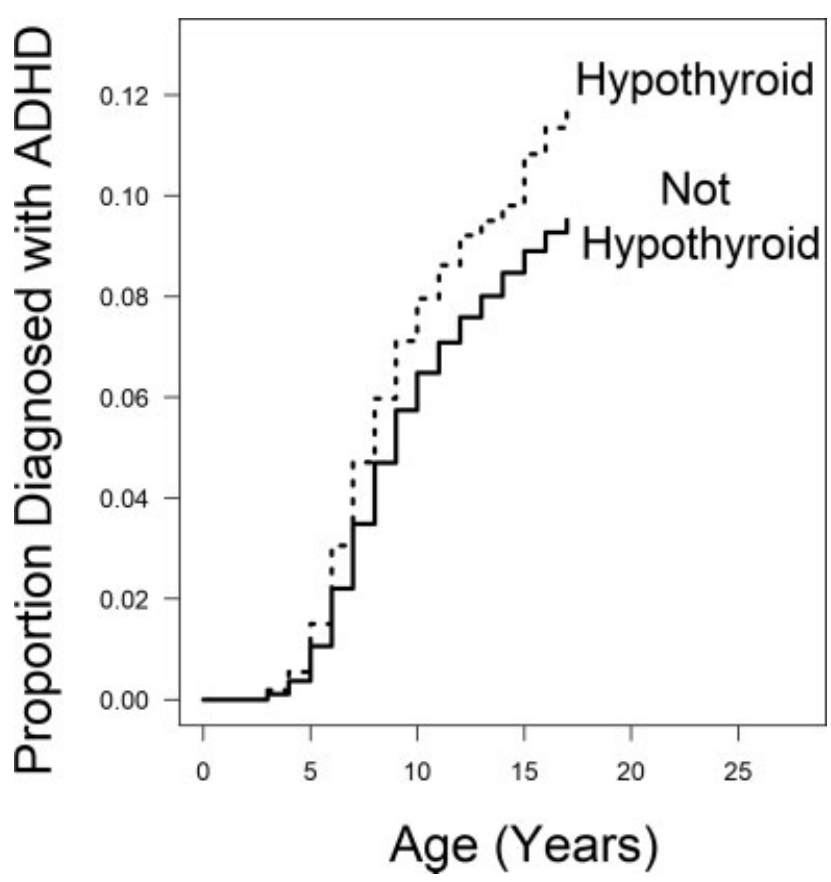

Fig. 2 Kaplan-Meier curves for attention-deficit hyperactivity disorder (ADHD) prevalence amongst children born to mothers with and without hypothyroidism in pregnancy. delivery with minimal effect on the magnitude of association. This suggests that preterm birth may not be in the causal pathway of ADHD but more likely an outcome independently associated with maternal hypothyroidism.

A stratified analysis based on race-ethnicity categories revealed that the effect of hypothyroidism was largest for Hispanic children. It is unlikely that this difference is due to racial ethnic differences in screening because of the standardized screening tools for both ADHD and maternal hypothyroidism that are in place across all KPSC centers. Further studies are needed to determine if there are genetic, dietary, or lifestyle factors that explain why children of Hispanic hypothyroid women develop ADHD at greater rates than other race-ethnicities. Although our adjusted proportional hazards models revealed some evidence for a statistical interaction, this could also be, in part, due to under sampling of some race ethnicities. Further studies with stratified sampling are needed to confirm our observations.

Our findings are largely consistent with previous studies linking thyroid dysfunction with neurodevelopmental disorders. In the generation R study, low concentrations of thyroxine ( $\leq 5$ th percentile) in pregnancy were associated with increased risk of teacher-reports of hyperactivity and inattention at 5 years of age. ${ }^{16} \mathrm{~A}$ follow-up study of this same patient population at 8 years of age also found that severe hypothyroidism during pregnancy significantly increases the risk of ADHD behaviors as rated by the parents. Although they adjusted for factors such as

Table 3 Association between maternal hypothyroidism and ADHD risk in the offspring based on gestational age at delivery and timing of first hypothyroidism diagnosis

\begin{tabular}{|c|c|c|c|c|c|c|c|}
\hline \multirow[t]{2}{*}{ Birth } & \multirow[t]{2}{*}{ Group } & \multirow[t]{2}{*}{$N$} & \multirow[t]{2}{*}{ Person-Years } & \multirow[t]{2}{*}{ IR (\%) } & \multirow[t]{2}{*}{ IRD (95\% CI) } & \multicolumn{2}{|c|}{ HR (95\% Confidence intervals) } \\
\hline & & & & & & Crude & Adjusted $^{\mathrm{a}}$ \\
\hline \multirow[t]{9}{*}{ Preterm } & No hypothyroidism & 1,515 & 218,583 & 6.93 & 0.00 (Reference) & 1.00 (Reference) & 1.00 (Reference) \\
\hline & Hypothyroidism & 70 & 7,640 & 9.16 & $2.23(0.32,4.14)$ & $1.38(1.09,1.76)$ & $1.33(1.04,1.69)$ \\
\hline & Pre-pregnancy & 54 & 5,407 & 9.99 & $3.06(0.80,5.31)$ & $1.50(1.15,1.97)$ & $1.43(1.09,1.88)$ \\
\hline & 120 to 61 days & 35 & 3,418 & 10.2 & $3.31(0.49,6.13)$ & $1.52(1.09,2.13)$ & $1.42(1.01,1.99)$ \\
\hline & 60 to 1 days & 19 & 1,989 & 9.55 & $2.62(-1.06,6.30)$ & $1.47(0.93,2.31)$ & $1.44(0.92,2.27)$ \\
\hline & In-pregnancy & 16 & 2,233 & 7.17 & $0.23(-3.23,3.70)$ & $1.09(0.66,1.78)$ & $1.07(0.66,1.76)$ \\
\hline & First trimester & 11 & 1,225 & 8.98 & $2.05(-2.63,6.73)$ & $1.39(0.77,2.52)$ & $1.40(0.77,2.54)$ \\
\hline & Second trimester & 5 & 549 & 9.11 & $2.18(-4.80,9.15)$ & $1.35(0.56,3.24)$ & $1.31(0.55,3.16)$ \\
\hline & Third trimester & 0 & 0 & 0.00 & ND & ND & ND \\
\hline \multirow[t]{9}{*}{ Term } & No hypothyroidism & 14,656 & $2,744,865$ & 5.34 & 0.00 (Reference) & 1.00 (Reference) & 1.00 (Reference) \\
\hline & Hypothyroidism & 455 & 72,620 & 6.27 & $0.93(0.39,1.47)$ & $1.23(1.12,1.35)$ & $1.22(1.11,1.34)$ \\
\hline & Pre-pregnancy & 323 & 50,385 & 6.41 & $1.07(0.43,1.72)$ & $1.26(1.13,1.40)$ & $1.24(1.11,1.38)$ \\
\hline & 120 to 61 days & 187 & 31,698 & 5.90 & $0.56(-0.25,1.37)$ & $1.16(1.01,1.34)$ & $1.15(0.99,1.33)$ \\
\hline & 60 to 1 days & 136 & 18,687 & 7.28 & $1.94(0.89,2.99)$ & $1.42(1.20,1.68)$ & $1.38(1.17,1.64)$ \\
\hline & In-pregnancy & 132 & 22,235 & 5.94 & $0.60(-0.37,1.56)$ & $1.17(0.99,1.39)$ & $1.18(0.99,1.40)$ \\
\hline & First trimester & 80 & 12,933 & 6.19 & $0.85(-0.42,2.11)$ & $1.27(1.02,1.58)$ & $1.26(1.01,1.57)$ \\
\hline & Second trimester & 22 & 4,773 & 4.62 & $-0.73(-2.80,1.34)$ & $0.87(0.57,1.32)$ & $0.87(0.57,1.32)$ \\
\hline & Third trimester & 30 & 4,529 & 5.90 & $1.28(-0.85,3.42)$ & $1.23(0.86,1.76)$ & $1.30(0.91,1.87)$ \\
\hline
\end{tabular}

Abbreviations: ADHD, attention-deficit hyperactivity disorder; $\mathrm{Cl}$, confidence intervals; $\mathrm{HR}$, hazard ratio; IR, incidence rate per 1,000 person-years; IRD, incidence rates difference; ND, not done due to small sample. Note: Statistically significant associations are indicated in boldface.

${ }^{a}$ Adjustments were made for maternal age, education, smoking during pregnancy, prenatal care, parity, median household income, child's sex, and race/ethnicity. 
Table 4 Effect of maternal hypothyroidism on incidence and hazard of childhood ADHD by child sex and timing of first hypothyroidism diagnosis

\begin{tabular}{|c|c|c|c|c|c|c|c|}
\hline \multirow{2}{*}{ Sex } & \multirow[b]{2}{*}{ Group } & \multirow[b]{2}{*}{$N$} & \multirow[b]{2}{*}{ Person-Years } & \multirow[b]{2}{*}{ IR (\%) } & \multirow[b]{2}{*}{ IRD (95\% Cl) } & \multicolumn{2}{|c|}{ HR (95\% Confidence intervals) } \\
\hline & & & & & & Crude & Adjusted $^{\mathrm{a}}$ \\
\hline \multirow[t]{9}{*}{ Female } & No hypothyroidism & 4,613 & $1,482,030$ & 3.11 & 0.00 (Reference) & 1.00 (Reference) & 1.00 (Reference) \\
\hline & Hypothyroidism & 148 & 41,159 & 3.60 & $0.48(-0.06,1.03)$ & $1.22(1.04,1.44)$ & $1.19(1.01,1.40)$ \\
\hline & Pre-pregnancy & 104 & 28,345 & 3.67 & $0.56(-0.10,1.21)$ & $1.24(1.02,1.51)$ & $1.20(0.99,1.46)$ \\
\hline & 120 to 61 days & 62 & 17,952 & 3.45 & $0.34(-0.48,1.16)$ & $1.17(0.91,1.51)$ & $1.13(0.88,1.45)$ \\
\hline & 60 to 1 days & 42 & 10,393 & 4.04 & $0.93(-0.15,2.00)$ & $1.36(1.01,1.85)$ & $1.32(0.97,1.44)$ \\
\hline & In-pregnancy & 44 & 12,814 & 3.43 & $0.32(-0.65,1.29)$ & $1.17(0.87,1.58)$ & $1.16(0.86,1.56)$ \\
\hline & First trimester & 27 & 7,364 & 3.67 & $0.55(-0.72,1.83)$ & $1.31(0.90,1.92)$ & $1.30(0.89,1.90)$ \\
\hline & Second trimester & 7 & 2,709 & 2.58 & $-0.53(-2.63,1.58)$ & $0.85(0.41,1.79)$ & $0.84(0.40,1.76)$ \\
\hline & Third trimester & 10 & 2,741 & 3.65 & $0.54(-1.55,2.62)$ & $1.15(0.62,2.13)$ & $1.11(0.60,2.07)$ \\
\hline \multirow[t]{9}{*}{ Male } & No hypothyroidism & 11,558 & $1,481,418$ & 7.80 & 0.00 (Reference) & 1.00 (Reference) & 1.00 (Reference) \\
\hline & Hypothyroidism & 377 & 39,101 & 9.64 & $1.84(0.95,2.73)$ & $1.30(1.17,1.44)$ & $1.26(1.14,1.40)$ \\
\hline & Pre-pregnancy & 273 & 27,447 & 9.95 & $2.14(1.09,3.20)$ & $1.34(1.18,1.51)$ & $1.30(1.15,1.46)$ \\
\hline & 120 to 61 days & 160 & 17,164 & 9.32 & $1.52(0.19,2.85)$ & $1.26(1.07,1.47)$ & $1.22(1.04,1.43)$ \\
\hline & 60 to 1 days & 113 & 10,283 & 10.99 & $3.19(1.47,4.90)$ & $1.47(1.22,1.77)$ & $1.43(1.19,1.72)$ \\
\hline & In-pregnancy & 104 & 11,654 & 8.92 & $1.12(-0.49,2.73)$ & $1.20(0.99,1.46)$ & $1.18(0.97,1.44)$ \\
\hline & First trimester & 64 & 6,794 & 9.42 & $1.62(-0.49,3.72)$ & $1.32(1.03,1.68)$ & $1.28(1.00,1.64)$ \\
\hline & Second trimester & 20 & 2,613 & 7.65 & $-0.15(-3.54,3.24)$ & $0.97(0.63,1.51)$ & $0.97(0.63,1.50)$ \\
\hline & Third trimester & 20 & 2,247 & 8.90 & $1.10(-2.56,4.76)$ & $1.16(0.75,1.80)$ & $1.16(0.75,1.81)$ \\
\hline
\end{tabular}

Abbreviations: $\mathrm{Cl}$, confidence interval; $\mathrm{HR}$, hazard ratio; IR, incidence rate per 1,000 person-years; IRD, incidence rate difference. Note: Statistically significant associations are indicated in boldface.

adjustments were made for maternal age, education, smoking during pregnancy, prenatal care, parity, median household income, and child's race/ethnicity.

child ethnicity, age, sex, and maternal demographics, no effects of these covariates on the association between maternal hypothyroidism and ADHD index in the children were reported. ${ }^{27} \mathrm{We}$ performed stratified analyses on a much larger sample size that relied on clinical diagnosis of both ADHD and maternal hypothyroidism. ${ }^{27}$ As in previous studies, ${ }^{28}$ we found that the effects of hypothyroidism on adverse neurodevelopmental outcomes were gestational age dependent. Our findings also differ from those of Andersen et al, who reported no association of maternal hypothyroidism with increased risk of ADHD in Denmark. ${ }^{18}$ This could be due to differences in timing of diagnosis or patient demographics. In nearly $80 \%$ of the pregnancies studied by Andersen et al, the thyroid dysfunction was diagnosed after the birth of the child; the vast majority of the Danish patient population is Caucasian. We found no effect of hypothyroidism diagnosed late in pregnancy on risk of childhood ADHD and that the effect was much stronger for Hispanics than other racesethnicities. There are large race/ethnic disparities in access to and utilization of health care in the United States and this may also contribute to undertreatment of hypothyroidisms and thus disparities in ADHD risk. This finding is consistent with our previous work that examined race-ethnicity as a risk modifier for autism in children born to women with hypothyroidism during pregnancy. ${ }^{29}$

ADHD may be one of several neurodevelopmental disorders that results from maternal hypothyroidism. Psychological testing of 7- to 9-year old children born to women with second-trimester TSH concentrations in the 98th percen- tile tended to have lower intelligence quotients (IQs), some language difficulties, higher rates of school problems, and to be more easily distracted ${ }^{19}$ than children born to mothers with normal TSH levels. ${ }^{19}$ Although we did not examine cognitive or motor function, we previously found higher rates of autism in children born to hypothyroid mothers in our patient population. ${ }^{29}$ Epilepsy, a common comorbidity in autism and ADHD, is also increased in children born to hypothyroid mothers. ${ }^{30}$

Hyperthyroidism may be equally disruptive to neurodevelopment. Our recent studies have demonstrated that hyperthyroidism increases the risk of autism. ${ }^{31}$ Furthermore, Hales et al, found that overtreatment of hypothyroidism in pregnant women increased the risk of ADHD in the offspring. ${ }^{32}$ This is consistent with previous studies that demonstrated a $U$-shaped curve between maternal thyroxine levels in pregnancy with childhood IQ. ${ }^{28}$ It is unclear from our study if the risk associated with higher levels of treatment reflect a toxic effects of treatment or a more severe form of the hypothyroidism.

Strengths of our study include, use of a large, well-maintained electronic database covering a large number of pregnancies over a long period of time, and measurements on the children with high follow-up. Consistent standards for diagnosing ADHD in all KPSC care settings minimize the risk for misclassification but may make it difficult to generalize our findings to other populations where diagnosis is made by a primary care provider. Ethnic diversity of the population studied as well as access to birth records and demographic 
Table 5 Effect of maternal hypothyroidism on incidence rate and risk of ADHD by child race-ethnicity and timing of first hypothyroidism diagnosis

\begin{tabular}{|c|c|c|c|c|c|c|c|}
\hline \multirow[b]{2}{*}{ Race-Ethnicity } & \multirow[b]{2}{*}{ Group } & \multirow[b]{2}{*}{$N$} & \multirow[b]{2}{*}{ Patient-Years } & \multirow[b]{2}{*}{ IR (\%) } & \multirow[b]{2}{*}{ IRD } & \multicolumn{2}{|c|}{ HR ( $95 \%$ confidence intervals) } \\
\hline & & & & & & Crude & Adjusted $^{\mathrm{a}}$ \\
\hline \multirow[t]{9}{*}{ NH-White } & No hypothyroidism & 4,131 & 531,076 & 7.78 & 0.000 (Reference) & 1.00 (Reference) & 1.00 (Reference) \\
\hline & Hypothyroidism & 191 & 21,162 & 9.03 & $1.25(0.03,2.46)$ & $1.21(1.05,1.40)$ & $1.22(1.05,1.41)$ \\
\hline & Pre-pregnancy & 151 & 15,776 & 9.57 & $1.79(0.39,3.19)$ & $1.28(1.09,1.51)$ & $1.29(1.09,1.52)$ \\
\hline & 120 to 61 days & 83 & 9,978 & 8.32 & $0.54(-1.21,2.28)$ & $1.11(0.89,1.38)$ & $1.12(0.90,1.39)$ \\
\hline & 60 to 1 days & 68 & 5,798 & 11.73 & $3.95(1.66,6.24)$ & $1.57(1.23,1.99)$ & $1.57(1.24,2.00)$ \\
\hline & In-pregnancy & 40 & 5,386 & 7.43 & $-0.35(-2.72,2.02)$ & $1.00(0.73,1.37)$ & $1.02(0.74,1.39)$ \\
\hline & First trimester & 28 & 3,316 & 8.44 & $0.66(-2.34,3.68)$ & $1.19(0.82,1.72)$ & $1.29(1.09,1.52)$ \\
\hline & Second trimester & 4 & 1,002 & 3.99 & $N^{c}$ & ND & ND \\
\hline & Third trimester & 8 & 1,068 & 7.49 & ND & ND & ND \\
\hline \multirow[t]{9}{*}{ Hispanic } & No hypothyroidism & 5,535 & $1,246,455$ & 4.44 & 0.00 (Reference) & 1.00 (Reference) & 1.00 (Reference) \\
\hline & Hypothyroidism & 182 & 30,118 & 6.04 & $1.60(0.84,2.37)$ & $1.43(1.23,1.66)$ & $1.45(1.25,1.68)$ \\
\hline & Pre-pregnancy & 117 & 20,027 & 5.84 & $1.40(0.47,2.33)$ & $1.38(1.15,1.65)$ & $1.39(1.15,1.67)$ \\
\hline & 120 to 61 days & 72 & 12,708 & 5.67 & $1.22(0.06,2.39)$ & $1.34(1.06,1.69)$ & $1.36(1.08,1.72)$ \\
\hline & 60 to 1 days & 45 & 7,319 & 6.15 & $1.71(0.18,3.24)$ & $1.44(1.07,1.93)$ & $1.42(1.06,1.92)$ \\
\hline & In-pregnancy & 65 & 10,091 & 6.44 & $2.00(0.69,3.31)$ & $1.53(1.20,1.95)$ & $1.59(1.24,2.03)$ \\
\hline & First trimester & 40 & 6,045 & 6.62 & $2.18(0.49,3.86)$ & $1.61(1.18,2.20)$ & $1.71(1.25,2.33)$ \\
\hline & Second trimester & 14 & 2,135 & 6.56 & $2.12(-0.71,4.94)$ & $1.48(0.88,2.50$ & $1.49(0.88,2.52)$ \\
\hline & Third trimester & 11 & 1,911 & 5.75 & $1.32(-1.67,4.30)$ & $1.33(0.74,2.40)$ & $1.35(0.75,2.44)$ \\
\hline \multirow[t]{6}{*}{$\mathrm{NH}-\mathrm{Black}^{\mathrm{b}}$} & No hypothyroidism & 1,481 & 232,596 & 6.37 & 0.00 (Reference) & 1.00 (Reference) & 1.00 (Reference) \\
\hline & Hypothyroidism & 19 & 2,623 & 7.24 & $0.88(-2.20,3.95)$ & $1.16(0.74,1.83)$ & $1.25(0.80,1.97)$ \\
\hline & Pre-pregnancy & 16 & 985 & 9.90 & $3.53(-0.38,7.45)$ & $1.57(0.96,2.58)$ & $1.64(1.00,2.68)$ \\
\hline & 120 to 61 days & 11 & 1,001 & 10.99 & $4.26(-0.34,9.58)$ & $1.74(0.96,3.15)$ & $1.79(0.99,3.25)$ \\
\hline & 60 to 1 days & 5 & 615 & 8.13 & ND & ND & ND \\
\hline & In-pregnancy & 3 & 1,007 & 2.98 & ND & ND & ND \\
\hline \multirow[t]{6}{*}{ Asian $/ \mathrm{PI}^{\mathrm{b}}$} & No hypothyroidism & 612 & 289,003 & 2.12 & 0.00 (Reference) & 1.00 (Reference) & 1.00 (Reference) \\
\hline & Hypothyroidism & 20 & 7,844 & 2.55 & $0.43(-0.60,1.47)$ & $1.28(0.82,2.00)$ & $1.32(0.35,2.07)$ \\
\hline & Pre-pregnancy & 16 & 5,544 & 2.89 & $0.77(-0.46,1.99)$ & $1.44(0.88,2.37)$ & $1.48(0.90,2.43)$ \\
\hline & 120 to 61 days & 8 & 3,477 & 2.30 & ND & ND & ND \\
\hline & 60 to 1 days & 8 & 2,067 & 3.87 & ND & ND & ND \\
\hline & In-pregnancy & 4 & 2,300 & 1.74 & ND & ND & ND \\
\hline \multirow[t]{6}{*}{ Other/Multiple ${ }^{\mathrm{b}}$} & No hypothyroidism & 4,399 & 454,344 & 6.65 & 0.00 (Reference) & 1.00 (Reference) & 1.00 (Reference) \\
\hline & Hypothyroidism & 113 & 18,461 & 6.35 & $-0.52(-1.72,0.67)$ & $0.96(0.80,1.16)$ & $1.02(0.84,1.23)$ \\
\hline & Pre-pregnancy & 77 & 12,790 & 6.02 & $-0.63(-2.05,0.80)$ & $0.94(0.75,1.18)$ & $1.03(0.82,1.29)$ \\
\hline & 120 to 61 days & 48 & 7,929 & 6.05 & $-0.59(-2.40,1.21)$ & $0.95(0.71,1.26)$ & $1.03(0.77,1.37)$ \\
\hline & 60 to 1 days & 29 & 4,861 & 5.97 & $-0.68(-2.98,1.62)$ & $0.93(0.65,1.34)$ & $1.02(0.71,1.48)$ \\
\hline & In-pregnancy & 36 & 5,671 & 6.35 & $-0.30(-2.43,1.84)$ & $1.00(0.72,1.39)$ & $0.99(0.71,1.38)$ \\
\hline
\end{tabular}

Abbreviations: ADHD, attention-deficit hyperactivity disorder; HR, hazard ratio; IR, incidence rate per 1,000 person-years; IRD, incidence rate difference; ND, not done; PI, Pacific Islander.

Note: Statistically significant associations are indicated in boldface.

${ }^{a}$ Adjustments were made for maternal age, education, smoking during pregnancy, prenatal care, parity, median household income, and child's sex.

bTrimester-specific data are not provided due to insufficient observation in the categories.

"Analysis "not done" due to insufficient observations in the categories.

information has further advantages. Although difficulties in diagnosing and treating minority children with ADHD are well-known, ${ }^{33}$ any differences in background risk for diagnosing ADHD would tend to bias the results of our study on hypothyroidism as a risk factor toward the null. By focusing our analysis on children with both a diagnosis and prescribed medications for ADHD, we further limited the possibilities of misclassification. Even with inclusion of these children in our sensitivity analyses, the effect size remained unchanged. Although we adjusted for several potential confounding factors, our findings are also limited by several residual factors that are inherent to retrospective epidemiological studies. 
Given the difficulty in accounting for all the comorbidities that may occur with ADHD in early preterm births, we excluded infants born at $<28$ weeks gestation. This limits our conclusions to moderately and late preterm birth. We also used strictly diagnostic codes. It is unclear if the hypothyroidism was due to autoimmune thyroid disease, thyroid cancers, Hashimoto's thyroiditis, environmental toxins, iodine deficiencies, or other causes of hypothyroidism. Although our findings are biologically plausible and the timing of the hypothyroidism precedes the development of ADHD, it is unclear if the relationship is dose-dependent and causal. Additional studies are also needed to determine the extent to which maternal hypothyroidism correlates with fetal hypothyroidism in our population. The placenta is rich in enzymes and other proteins that regulate iodine and thyroid hormone concentrations at the maternal-fetal interface. ${ }^{21}$ It is possible that fetal hypothyroidism occurs in the absence of maternal hypothyroidism or vice versa. We are also unable to explore what specific changes that thyroid hormone deficiencies cause in the fetal brain to result in ADHD and how they may differ from other neurodevelopmental disorders associated with maternal hypothyroidism.

In summary, maternal hypothyroidism during the preconceptual period and first trimester significantly increases the risk of ADHD in children. Further studies are needed to identify different types of hypothyroidism that is contributing to the increased risk and to better understand the molecular mechanisms behind them.

\section{Conflict of Interest}

None declared.

\section{References}

1 Mahone EM, Denckla MB. Attention-deficit/hyperactivity disorder: a historical neuropsychological perspective. J Int Neuropsychol Soc 2017;23(9-10):916-929

2 Austerman J. ADHD and behavioral disorders: Assessment, management, and an update from DSM-5. Cleve Clin J Med 2015;82 (11, Suppl 1):S2-S7

3 Mannuzza S, Klein RG, Bessler A, Malloy P, LaPadula M. Adult outcome of hyperactive boys. Educational achievement, occupational rank, and psychiatric status. Arch Gen Psychiatry 1993;50 (07):565-576

4 Semiz UB, Basoglu C, Oner O, et al. Effects of diagnostic comorbidity and dimensional symptoms of attention-deficit-hyperactivity disorder in men with antisocial personality disorder. Aust $\mathrm{N}$ Z J Psychiatry 2008;42(05):405-413

5 Murphy K, Barkley RA. Attention deficit hyperactivity disorder adults: comorbidities and adaptive impairments. Compr Psychiatry 1996;37(06):393-401

6 Gray SA, Fettes P, Woltering S, Mawjee K, Tannock R. Symptom manifestation and impairments in college students with ADHD. J Learn Disabil 2016;49(06):616-630

7 Koisaari T, Michelsson K, Holopainen JM, et al. Traffic and criminal behavior of adults with attention deficit-hyperactivity with a prospective follow-up from birth to the age of 40 years. Traffic Inj Prev 2015;16(08):824-830

8 Furczyk K, Thome J. Adult ADHD and suicide. Atten Defic Hyperact Disord 2014;6(03):153-158

9 DoshiJA, Hodgkins P, Kahle J, et al. Economic impact of childhood and adult attention-deficit/hyperactivity disorder in the United States. J Am Acad Child Adolesc Psychiatry 2012;51(10):990-1002.e2
10 Kim JS, Bailey MJ, Weller JL, et al. Thyroid hormone and adrenergic signaling interact to control pineal expression of the dopamine receptor D4 gene (Drd4). Mol Cell Endocrinol 2010;314(01): 128-135

11 Evans IM, Sinha AK, Pickard MR, Edwards PR, Leonard AJ, Ekins RP. Maternal hypothyroxinemia disrupts neurotransmitter metabolic enzymes in developing brain. J Endocrinol 1999;161(02): 273-279

12 Cvijić G, Petrović N, Djordjević J, Davidović V, Petrović VM. Effect of cold exposure on serum DBH and interscapular brown adipose tissue MAO activity in hypothyroid T3- and T4-treated rats. Ann N Y Acad Sci 2004;1018:214-218

13 Atıci E, Mogulkoc R, Baltaci AK, Menevse E. The effect of thyroid dysfunction on nesfatin-1 and adiponectin levels in rats. Horm Mol Biol Clin Investig 2017;32(03):/j/hmbci.2017.32.issue-3/ hmbci-2017-0033/hmbci-2017-0033.xml

14 Shafiee SM, Vafaei AA, Rashidy-Pour A. Effects of maternal hypothyroidism during pregnancy on learning, memory and hippocampal BDNF in rat pups: Beneficial effects of exercise. Neuroscience 2016;329:151-161

15 Ghassabian A, Bongers-Schokking JJ, de Rijke YB, et al. Maternal thyroid autoimmunity during pregnancy and the risk of attention deficit/hyperactivity problems in children: the Generation $R$ study. Thyroid 2012;22(02):178-186

16 Oostenbroek MHW, Kersten RHJ, Tros B, Kunst AE, Vrijkotte TGM, Finken MJJ. Maternal hypothyroxinaemia in early pregnancy and problem behavior in 5-year-old offspring. Psychoneuroendocrinology 2017;81:29-35

17 Päkkilä $\mathrm{F}$, Männistö $\mathrm{T}$, Pouta $\mathrm{A}$, et al. The impact of gestational thyroid hormone concentrations on ADHD symptoms of the child. J Clin Endocrinol Metab 2014;99(01):E1-E8

18 Andersen SL, Laurberg P, Wu CS, Olsen J. Attention deficit hyperactivity disorder and autism spectrum disorder in children born to mothers with thyroid dysfunction: a Danish nationwide cohort study. BJOG 2014;121(11):1365-1374

19 Haddow JE, Palomaki GE, Allan WC, et al. Maternal thyroid deficiency during pregnancy and subsequent neuropsychological development of the child. N Engl J Med 1999;341(08):549-555

20 Sahai H, Khurshid A. Statistics in Epidemiology: Methods, Techniques and Applications. Boca Raton, FL: CRC Press LLC; 1996

21 Landers K, Richard K. Traversing barriers-how thyroid hormones pass placental, blood-brain and blood-cerebrospinal fluid barriers. Mol Cell Endocrinol 2017;458:22-28

22 Lindström K, Lindblad F, Hjern A. Preterm birth and attention-deficit/hyperactivity disorder in schoolchildren. Pediatrics 2011; 127(05):858-865

23 Perricone G, Morales MR, Anzalone G. Neurodevelopmental outcomes of moderately preterm birth: precursors of attention deficit hyperactivity disorder at preschool age. Springerplus 2013;2(01):221

24 Liu YY, Brent GA. Thyroid hormone and the brain: mechanisms of action in development and role in protection and promotion of recovery after brain injury. Pharmacol Ther 2018;186:176-185

25 Sheehan PM, Nankervis A, Araujo Júnior E, Da Silva Costa F. Maternal thyroid disease and preterm birth: systematic review and metaanalysis. J Clin Endocrinol Metab 2015;100(11):4325-4331

26 Andersen SL, Olsen J, Wu CS, Laurberg P. Low birth weight in children born to mothers with hyperthyroidism and high birth weight in hypothyroidism, whereas preterm birth is common in both conditions: a Danish National Hospital Register study. Eur Thyroid J 2013;2(02):135-144

27 Modesto T, Tiemeier H, Peeters RP, et al. Maternal mild thyroid hormone insufficiency in early pregnancy and attention-deficit/hyperactivity disorder symptoms in children. JAMA Pediatr 2015;169(09):838-845

28 Korevaar TI, Muetzel R, Medici M, et al. Association of maternal thyroid function during early pregnancy with offspring IQ and brain morphology in childhood: a population-based prospective cohort study. Lancet Diabetes Endocrinol 2016;4(01):35-43 
29 Getahun D, Jacobsen SJ, Fassett MJ, et al. Association between maternal hypothyroidism and autism spectrum disorders in children. Pediatr Res 2018;83(03):580-588

30 Andersen SL, Laurberg P, Wu CS, Olsen J. Maternal thyroid dysfunction and risk of seizure in the child: a Danish nationwide cohort study. J Pregnancy 2013;2013:636705

31 Getahun D, Fassett MJ, Chiu VY, Peltier MR. Maternal hyperthyroidism increases the risk of autism spectrum disorders in the offspring.
Paper presented at: Society for Reproductive Investigation (SRI) 67th Annual Scientific Meeting S-089 March 13, 2020; 2020

32 Hales C, Taylor PN, Channon S, et al. Controlled antenatal thyroid screening II: effect of treating maternal suboptimal thyroid function on child behavior. J Clin Endocrinol Metab 2020;105(03):dgz098

33 Miller TW, Nigg JT, Miller RL. Attention deficit hyperactivity disorder in African American children: what can be concluded from the past ten years? Clin Psychol Rev 2009;29(01):77-86 